\section{Cognitive Processing of Weight and Emotional Information in Disordered Eating}

Current Directions in Psychological

Science

19(2) 81-85

(C) The Author(s) 2010

Reprints and permission:

sagepub.com/journalsPermissions.nav DOI: I0.1 I77/096372|410364007

http://cdps.sagepub.com

(SAGE

\author{
Teresa A. Treat' and Richard J. Viken ${ }^{2}$ \\ ${ }^{\prime}$ Yale University and ${ }^{2}$ Indiana University
}

\begin{abstract}
Numerous theories propose a role for cognitive processing in disordered eating, but clinical scientists have yet to capitalize fully on the wealth of approaches developed by quantitative cognitive scientists when evaluating such theories. This article describes research that uses well-established and theoretically rich cognitive science approaches to demonstrate that women who struggle with disordered eating, relative to their peers, exhibit differential processing of other women's emotional and weight information presented in photo stimuli. More generally, this work illustrates the portability of cognitive science approaches to the study of clinically relevant individual differences in complex, socially relevant processing.
\end{abstract}

\title{
Keywords
}

eating disorders, attention, memory, covariation detection, information processing

Large numbers of young women struggle every day with symptoms of eating disorders in an effort to achieve a glamorized ideal of thinness (American Psychiatric Association, 2006). Some binge, some restrict, some purge, some exercise excessively, and some evaluate their personal worth almost exclusively on the basis of their shape and weight. In an effort to understand and treat these serious behavioral health problems, researchers increasingly have focused on the role of cognitive factors, such as distorted processing of shape and weight, in the development and maintenance of disordered eating and in the evolution of cognitive-behavioral treatments (e.g., Fairburn, Cooper, \& Shafran, 2003). For example, Vitousek (1996) proposed that attention to and memory for shape-, weight-, and eating-related information play a central role in disordered eating, such that women who struggle with disordered eating might focus more on and better remember weight-related information that is present in their social environments. Lee and Shafran (2004), in a recent literature review, concluded that treatment-seeking women may show enhanced attention to and memory for such information, consistent with information-processing models of eating disorders. Further investigation of the role of cognitive processes in disordered eating would benefit from greater attention to advances in cognitive science, however. Researchers in this area have developed a much wider array of theories, paradigms, and analytic strategies than currently are being employed, and they would seem to be promising for advancing cognitive theories of eating disorders (Treat et al., 2007).
Thus, the research described in this article draws on contemporary cognitive science to evaluate whether women who report eating-disorder symptoms, when compared with nonsymptomatic women, exhibit differential processing of other women's emotional and weight information. The approaches discussed are theoretically rich, highly integrated, and well grounded in quantitative cognitive science. These approaches require adaptation to study clinical questions, however, because of two fundamental differences between cognitive and clinical science. First, cognitive scientists typically develop formal models of what is shared across people in the operation of cognitive processes, whereas clinical scientists more commonly study individual differences in processing. Second, cognitive scientists frequently rely on simple, artificial stimuli, whereas clinical scientists often study processing of more complex, socially and emotionally relevant stimuli. Thus, at the most general level, the research described here illustrates the feasibility and utility of adopting a hybrid clinical-cognitive approach to the examination of clinical problems, using the example of cognitive factors in disordered eating.

\section{Corresponding Author:}

Address correspondence to Teresa A. Treat, Departments of Psychology and Cognitive Science, Yale University, PO Box 208205, New Haven, CT 06520-8205

E-mail: teresa.treat@yale.edu 


\section{Attention and Memory Processes in Disordered Eating}

Our first study examined the link between self-reported disordered eating and attention to other women's body-size and facial-affect information among undergraduate women (Viken, Treat, Nosofsky, McFall, \& Palmeri, 2002). We developed a photo stimulus set of 24 young women who varied along two dimensions: facial affect (happy to sad) and body size (heavy to light). The use of photos allowed us to investigate the relevance of participants' processing of other women's characteristics. This is a surprisingly understudied area of research, given the importance of socialcomparison processes in disordered eating (e.g., Cash, 2002). We chose facial affect as the second dimension because affective perception and memory are central to effective social interactions and management of emotions. Moreover, many women with eating disorders display deficits in interpersonal problem solving and indicate that negative mood frequently triggers eating-disordered behaviors (e.g., Smyth et al., 2007). Thus, we anticipated that highsymptom women, relative to low-symptom women, would display enhanced attention to body-size information and decreased attention to affective information.

Participants completed two tasks commonly used by cognitive scientists to quantify differential attention. In the similarity ratings task, participants judged the similarity of all possible pairs of the 24 photos. Similarity ratings provide rich information about individual differences in attentional focus. For example, a participant who attends little to other women's happiness might view a happy woman and a sad woman as very similar if both are thin, whereas a person who attends a great deal to other women's emotions might view these two women as very different. Multidimensional scaling procedures can be used to estimate each participant's attention to body-size and affect information.

In the prototype classification task, participants viewed two prototypical women who varied along body-size and affect dimensions (e.g., a "Type D woman," who was viewed by a sample of undergraduate women as sad and thin, and a "Type K woman," who was viewed as happy and heavy; the prototype labels were arbitrarily chosen letters). Participants then classified each remaining woman as more similar to a Type D or Type K woman. These classification judgments also tell us about the focus of attention. For example, a participant who classifies a sad, heavy woman as a Type D woman is relying more on affect, whereas a participant who classifies her as a Type $\mathrm{K}$ woman is relying more on body size. After collecting classification judgments across multiple sets of prototypes, we can use multilevel regression techniques to estimate how much each participant attends to or uses affect, relative to body-size, information.

As predicted, high-symptom women, relative to low-symptom women, showed greater attention to body-size information and less attention to affect information on the similarity ratings task, as well as greater relative attention to body-size than affect information on the prototype classification task. Enhanced relative processing of body size among high-symptom women is fully consistent with cognitive models of eating disorders. The symptom-linked differences in relative attention to affect suggest that disordered eating may be maintained by both enhanced processing of shape- and weight-related information and impoverished processing of affective information. These findings also highlight the importance of considering processing of information about other people, not just about oneself.

Recently, we evaluated the extent to which attention, memory, and correlation-detection processes operate differently among high-, medium-, and low-symptom college-aged women (Treat, Viken, Kruschke, \& McFall, in press). Participants first completed the prototype classification task with 28 photo stimuli, followed by a recognition memory task. The memory task presented both the 28 previously viewed photos and 28 new photos of the same women with either altered affect or altered body size (e.g., a previously light woman was modified using warping software to appear heavy). Participants then judged whether they had seen the exact photo previously. Formal modeling techniques were used to estimate memory for affect and for body size for each participant.

As expected, in the prototype classification task, highsymptom participants attended relatively more to body size than affect, when compared with medium- and low-symptom participants (see Fig. 1a). This finding extended our earlier work, which examined only high- and low-symptom women. High-symptom women also showed better memory for bodysize information than did low-symptom women and worse memory for affective information than did medium- and lowsymptom women (see Fig. 1b). These findings presumably reflect, at least in part, participants' differential relative attention to body-size and affect information. The memory deficit for affective information among high-symptom women is of particular theoretical interest, given the constraints this might place on interpersonal effectiveness.

\section{Correlation-Detection Processes in Disordered Eating}

Research has demonstrated (a) that young women who exhibit disordered eating endorse strong beliefs about the links between body size and a variety of indicators of happiness (e.g., being thin is associated with being happy) and (b) that stronger expectations about the negative association between weight and global self-improvement (i.e., heavier weight is linked to less happiness) predict the onset and worsening of disordered eating among adolescent girls (e.g., Hohlstein, Smith, \& Atlas, 1998; Smith, Simmons, Flory, Annus, \& Hill, 2007). This suggests that high-symptom women might perceive a negative association between other women's weight and happiness even when no such relationship is presentcommonly referred to as an illusory correlation. Within the realm of anxiety, numerous researchers have demonstrated that clinically anxious persons perceive illusory correlations between feared stimuli and negative outcomes (e.g., Tomarken, Mineka, \& Cook, 1989). In these studies, participants perceive that feared stimuli (e.g., snakes) are associated with aversive outcomes (e.g., shocks), even though the relationship between 


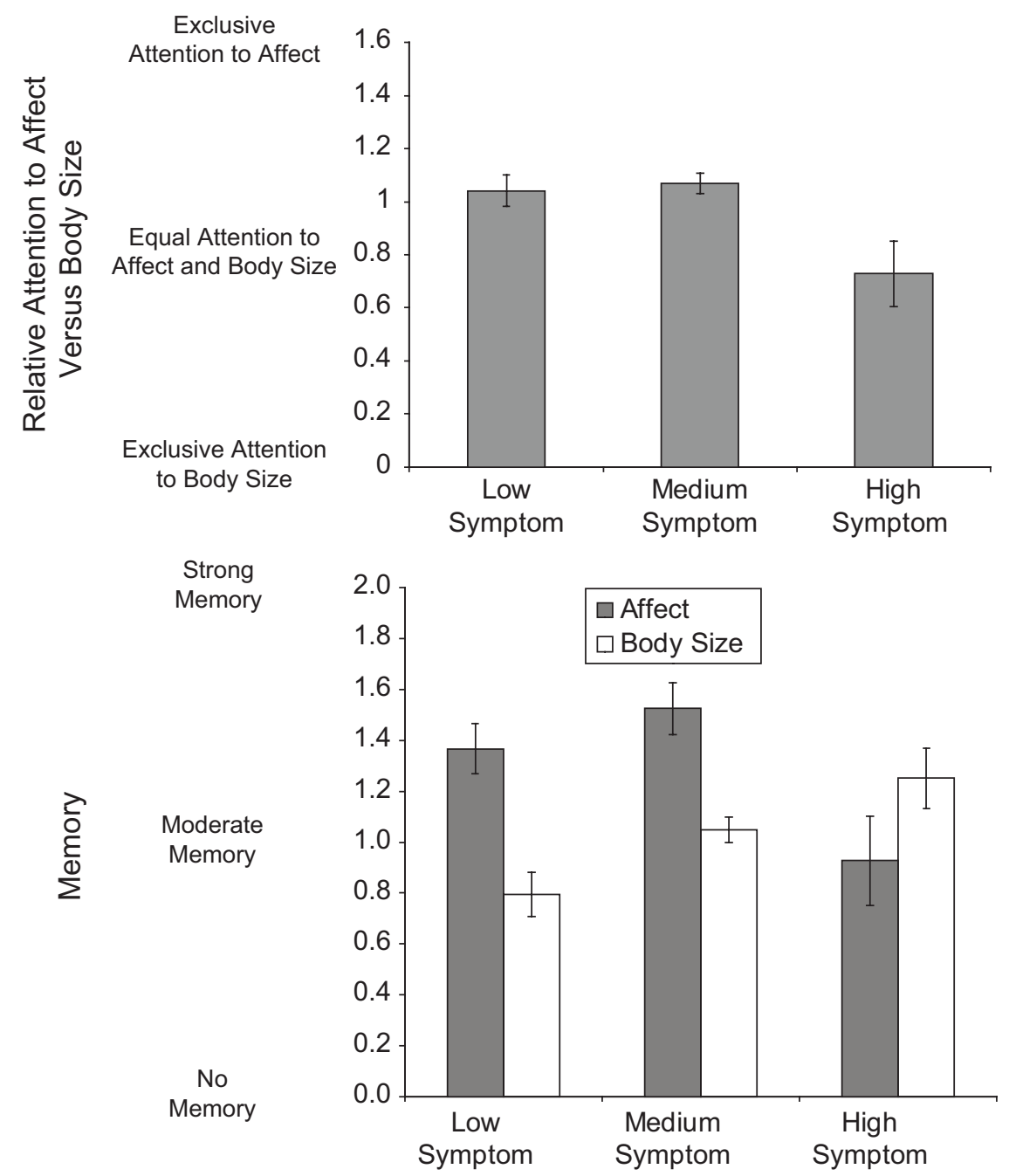

Fig. I. Relative attention to affect versus body size among high-, medium-, and lowsymptom participants on the prototype classification task (top); and memory for affect and body size among high-, medium-, and low-symptom participants (bottom).

stimuli and outcomes is random (e.g., a snake is just as likely to be paired with a pleasant tone as a shock). Such illusory correlations presumably encourage irrational fears about threatrelevant information. Similarly, perceiving an illusory negative correlation between other women's body size and affect (i.e., heavier body size is linked to less positive affect) might validate and intensify the weight-related concerns of highsymptom women (see Viken, Treat, Bloom, \& McFall, 2005).

Cognitive, social, and clinical scientists who study illusory correlation commonly expose participants only to stimulus conditions in which there is no relationship between the studied dimensions, stimuli, or events. The real world presents a much wider range of correlations, however. Contexts that glamorize thinness present a very strong negative correlation, for example, in which happiness is tied closely to being thin. More real-world contexts present far weaker associations, in which thinner women sometimes fail and heavier women sometimes succeed. Little is known about clinically relevant individual differences in sensitivity to varying levels of associations (i.e., how well persons discriminate correlations of different magnitude), as clinical scientists typically study perception of only random associations. Thus, we explored the possibility that sensitivity might vary among high-, medium-, and lowsymptom women.

We developed a correlation-detection task to examine whether high-symptom women showed a stronger illusory correlation than did low-symptom women, as well as altered sensitivity (Treat et al., in press; see Crump, Hannah, Allan, \& Hord, 2007, for a related paradigm). On each 8-second trial, participants viewed a rapidly presented series of 16 women for 500 milliseconds apiece and then judged the correlation of the women's affect and body size. On a trial depicting a perfect negative correlation, for example, all 16 women were either happy and thin or sad and heavy, consistent with stereotypical beliefs about the connection between body size and affect in our culture. In contrast, a trial presenting a perfectly positive correlation displayed 16 women who were either happy and heavy or sad and thin. And on a trial displaying no correlation, 


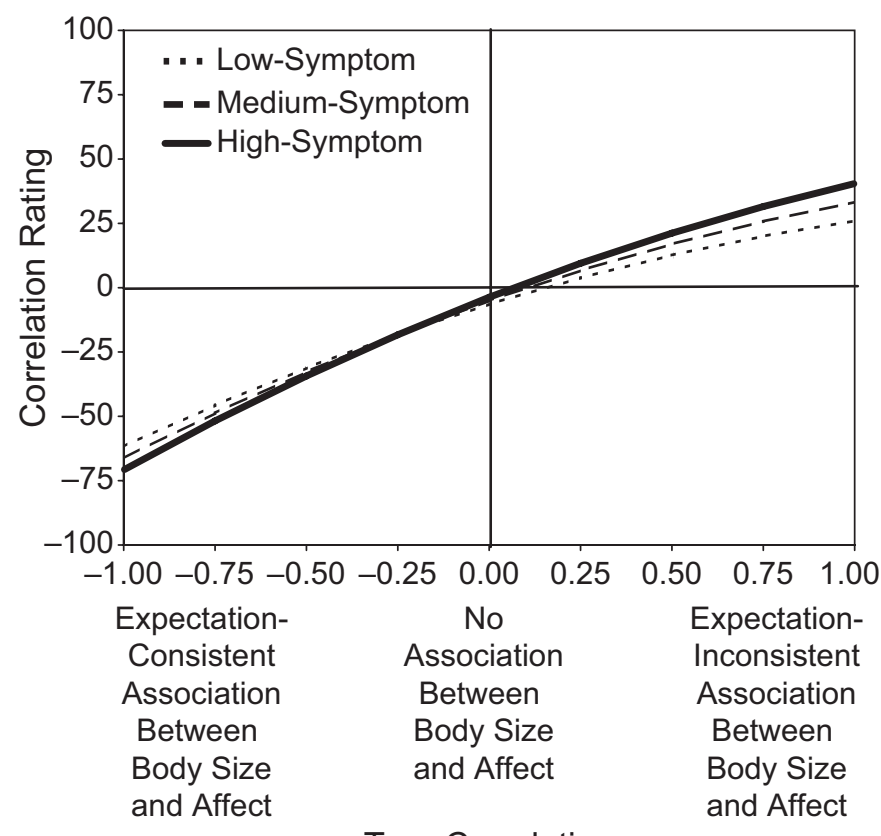

True Correlation

Fig. 2. Model-predicted performance on the covariation detection task among high-, medium-, and low-symptom participants. The true correlations presented to participants ranged from a perfectly negative $(-I .0)$ to a perfectly positive $(+I .0)$ association between women's affect and body size; participants' ratings of these correlations could range from perfectly negative $(-100)$ to perfectly positive $(+100)$.

one quarter of the 16 women were happy and thin, one quarter were happy and heavy, one quarter were sad and thin, and one quarter were sad and heavy. Nine trials were presented to each participant in a random order, and each trial presented a different level of correlation. The correlation rating scale ranged from -100 (perfectly negative correlation) to 0 (no correlation) to +100 (perfectly positive correlation). A statistical approach based on signal-detection theory was used to estimate the magnitude of each participant's illusory correlation and sensitivity.

Figure 2 depicts the results for the high-, medium-, and lowsymptom women based on the statistical model. The true correlation ranges on the $\mathrm{X}$-axis from -1 (expectation-consistent association between body size and affect) to +1 (expectationinconsistent association). The correlation rating on the $\mathrm{Y}$-axis ranges from -100 to +100 , as described above. To facilitate interpretation, we have superimposed two lines on this figure; the vertical line corresponds to a true correlation of zero and the horizontal line indexes a correlation rating of zero. Overall, participants displayed an illusory correlation in the belief-consistent direction, whereby thin women were more likely to be happy, even when there was no connection between thinness and happiness. You can see this in Figure 2 by noting that each group's predicted correlation rating is negative when the true correlation is zero (i.e., the predicted ratings for all three groups fall below zero (the horizontal line) when the true correlation is zero (the vertical line). Interestingly, high-, medium-, and low-symptom women did not differ in the strength of their illusory correlations (i.e., the predicted correlation rating when the true correlation is zero is very similar across groups).

Participants also showed significant sensitivity (ability to distinguish different levels of correlation) to the manipulated association. Sensitivity is represented in Figure 2 by the extent to which each group's predicted correlation ratings increased as the true correlation increased. All three groups' correlation ratings increased as the true correlation increased, particularly when the true correlation was in the stereotypically consistent direction. In other words, participants better distinguished trials consistent with their expectations (i.e., in which thinness predicted happiness) than trials inconsistent with their expectations (i.e., in which thinness predicted sadness). Note that the groups differed in how sensitive they were to the manipulated correlation. In particular, high-symptom women showed the strongest sensitivity, and low-symptom participants the weakest (i.e., high-symptom women's correlation ratings varied more as a function of the true correlation).

Ironically, such enhanced sensitivity might be expected to work against high-symptom participants to the extent that society presents them with far more social environments with negative correlations between weight and indicators of success. In other words, if high-symptom women are more sensitive to the connection between weight and happiness, and if most of the information they get about this relationship suggests that thinness predicts positive outcomes, then they might conclude that the "true" association is stronger than it is in reality. Research also indicates that high-symptom women view more mass media that idealize thinness than their peers do. Thus, one possibility is that both society- and individual-based greater exposure to social environments presenting negative associations increases risk for eating disorders, by providing more opportunities to cement perception of a negative association between weight and happiness. On the one hand, the entrenched nature of these perceptions may constrain high-symptom women's responsiveness to intervention strategies. On the other hand, however, high-symptom women's enhanced sensitivity may position them well to benefit from cognitive training interventions designed to challenge unrealistic beliefs about the link between weight and positive social and emotional outcomes.

\section{Conclusions}

The research described above has demonstrated that collegeaged women who report disordered eating, in comparison to their peers, show relatively greater attention to body size than to affect, worse memory for affect, better memory for body size, and superior sensitivity to the association between body size and affect. Notably, although depression commonly co-occurs with disordered eating, all observed group differences were specific to disordered eating. Future work should examine whether the processing patterns are more pronounced among treatment-seeking women and are present prior to symptom onset or develop only in conjunction with or subsequent to illness. It also will be important to investigate whether the processing patterns can be modified via experimental 
training methods or other interventions, such as cognitive behavioral therapy. Ultimately, the true promise of integrative clinical-cognitive science will lie in the development of novel approaches to reduce further the burden of disordered eating.

Most existing cognitive theories of eating disorders focus on attention to and memory for one's own shape- and weightrelated information. Thus, they might be extended by incorporating a more explicit role for processing of information about others in addition to oneself. Greater consideration of the role of emotional processing also appears warranted, as deficits in attention to and memory for another's affect might account in part for the common social and emotional difficulties in this population.

More generally, the presented work illustrates what hybrid clinical-cognitive science has to offer (a) clinical scientists' efforts to understand and modify processing patterns linked to psychopathology; and (b) cognitive scientists' efforts to evaluate the generalizability of their approaches to more complex, socially relevant circumstances (e.g., Lee \& Webb, 2005). A growing body of research attests to the portability of cognitive science approaches to examinations of clinically relevant processing (e.g., Farris, Viken, \& Treat, in press; Neufeld, 2007; Treat et al., in press; Viken et al., 2002). Thus, the time is ripe for the rapid exploration of the relatively unmapped region of quantitative clinical-cognitive science.

\section{Recommended Reading}

Neufeld, R.W.J. (2007). (See References). An edited volume containing numerous examples of the application of quantitative cognitive science to clinically relevant questions.

Neufeld, R.W.J. (in press). Contribution of mathematical psychology to psychological clinical science and assessment: Special Issue. Journal of Mathematical Psychology. A collection of papers providing an up-to-date overview of the application of cognitive science to a wide variety of clinically relevant questions.

Treat, T.A., McFall, R.M., Viken, R.J., Kruschke, J.K., Nosofsky, R.M., \& Wang, S.S. (2007). (See References). A paper providing a full discussion of the application of quantitative cognitive science to the study of clinically relevant processing of socially and emotionally relevant stimuli.

Treat, T.A., Viken, R.J., Kruschke, J.K., \& McFall, R.M. (in press). (See References). A paper discussing the role of attention, memory, and correlation-detection processes in disordered eating in more detail than the current article.

Viken, R.J., Treat, T.A., Nosofsky, R.M., McFall, R.M., \& Palmeri, T. (2002). (See References). A representative study illustrating original research about the operation of attentional processes in disordered eating.

\section{Declaration of Conflicting Interests}

The authors declared that they had no conflicts of interest with respect to their authorship or the publication of this article.

\section{References}

American Psychiatric Association. (2006). Treatment of patients with eating disorders, third edition. American Journal of Psychiatry, 163(Suppl. 7), 4-54.

Cash, T.F. (2002). Women's body images. In G.M. Wingood \& R.J. DiClemente (Eds.), Handbook of women's sexual and reproductive health. Issues in women health (pp. 175-194). New York: Kluwer Academic/Plenum Publishers.

Crump, M.J.C., Hannah, S.D., Allan, L.G., \& Hord, L.K. (2007). Contingency judgements on the fly. The Quarterly Journal of Experimental Psychology, 60, 753-763.

Fairburn, C.G., Cooper, Z., \& Shafran, R. (2003). Cognitive behaviour therapy for eating disorders: A "transdiagnostic" theory and treatment. Behaviour Research and Therapy, 41, 509-528.

Farris, C.A., Viken, R.J., \& Treat, T.A. (in press). Perceived association between diagnostic and non-diagnostic cues of women's sexual interest: General recognition theory predictors of risk for sexual coercion. Journal of Mathematical Psychology.

Hohlstein, L.A., Smith, G.T., \& Atlas, J.G. (1998). An application of expectancy theory to eating disorders: Development and validation of measures of eating and dieting expectancies. Psychological Assessment, 10, 49-58.

Lee, M., \& Shafran, R. (2004). Information processing biases in eating disorders. Clinical Psychology Review, 24, 215-238.

Lee, M.D., \& Webb, M.R. (2005). Modeling individual differences in cognition. Psychonomic Bulletin \& Review, 12, 605-621.

Neufeld, R.W.J. (2007). Advances in clinical-cognitive science: Formal modeling and assessment of processes and symptoms. Washington, DC: APA Books.

Smith, G.T., Simmons, J.R., Flory, K., Annus, A.M., \& Hill, K.K. (2007). Thinness and eating expectancies predict subsequent binge-eating and purging behavior among adolescent girls. Journal of Abnormal Psychology, 116, 188-197.

Smyth, J.M., Wonderlich, S.A., Heron, K.E., Sliwinski, M.J., Crosby, R.D., Mitchell, J.E., \& Engel, S.G. (2007). Daily and momentary mood and stress are associated with binge eating and vomiting in bulimia nervosa patients in the natural environment. Journal of Consulting and Clinical Psychology, 75, 629-638.

Tomarken, A.J., Mineka, S., \& Cook, M. (1989). Fear-relevant selective associations and covariation bias. Journal of Abnormal Psychology, 98, 381-394.

Treat, T.A., McFall, R.M., Viken, R.J., Kruschke, J.K., Nosofsky, R.M., \& Wang, S.S. (2007). Clinical-cognitive science: Applying quantitative models of cognitive processing to examination of cognitive aspects of psychopathology. In R.W.J. Neufeld (Ed.), Advances in clinical-cognitive science: Formal modeling and assessment of processes and symptoms (pp. 179205). Washington, DC: APA Books.

Treat, T.A., Viken, R.J., Kruschke, J.K., \& McFall, R.M. (in press). Role of attention, memory, and covariation-detection processes in clinically significant eating-disorder symptoms. Journal of Mathematical Psychology.

Viken, R.J., Treat, T.A., Bloom, S.L., \& McFall, R.M. (2005). Illusory correlation for body type and happiness: Covariation bias and its relationship to eating disorder symptoms. International Journal of Eating Disorders, 38, 65-72.

Viken, R.J., Treat, T.A., Nosofsky, R.M., McFall, R.M., \& Palmeri, T. (2002). Bulimics and controls' differential attention to and classification of body-size and affect stimulus information. Journal of Abnormal Psychology, 111, 598-609.

Vitousek, K.M. (1996). The current status of cognitive-behavioral models of anorexia nervosa and bulimia nervosa. In P.M. Salkovskis (Ed.), Frontiers of cognitive therapy (pp. 383-418). New York: Guilford Press. 